Bull. Korean Math. Soc. 49 (2012), No. 3, pp. 655-667

http://dx.doi.org/10.4134/BKMS.2012.49.3.655

\title{
CRITICAL POINT METRICS OF THE TOTAL SCALAR CURVATURE
}

\author{
Jeongwook Chang, Seungsu Hwang, and Gabjin Yun
}

\begin{abstract}
In this paper, we deal with a critical point metric of the total scalar curvature on a compact manifold $M$. We prove that if the critical point metric has parallel Ricci tensor, then the manifold is isometric to a standard sphere. Moreover, we show that if an $n$-dimensional Riemannian manifold is a warped product, or has harmonic curvature with non-parallel Ricci tensor, then it cannot be a critical point metric.
\end{abstract}

\section{Introduction}

Let $M$ be an $n$-dimensional compact manifold and $\mathcal{M}$ be the space of $C^{\infty}$ Riemannian metrics on $M$. Let $\mathcal{M}_{1} \subset \mathcal{M}$ be the set of smooth Riemannian structures on $M$ of volume 1 . The total scalar curvature functional $\mathcal{S}: \mathcal{M}_{1} \rightarrow \mathbb{R}$ is defined by

$$
\mathcal{S}(g)=\int_{M^{n}} s_{g} d v_{g}
$$

Einstein and Hilbert showed that critical points of $\mathcal{S}$ are Einstein metrics. In other words, the critical points satisfy $z_{g}=0$, where $z_{g}$ is the traceless Ricci tensor. Due to the resolution of Yamabe problem, we may consider a non-empty set

$$
\mathcal{C}=\left\{g \in \mathcal{M}_{1} \mid s_{g} \text { constant }\right\} .
$$

In 1987 Besse proposed a conjecture that the critical points of the total scalar curvature $\mathcal{S}$ restricted to $\mathcal{C}$ are Einstein metrics (see [1], p. 128). We will call it Conjecture I throughout the paper. The Euler-Lagrange equation for a critical point $g$ of this restricted variational problem can be written as the following

Received March 16, 2011; Revised May 18, 2011.

2010 Mathematics Subject Classification. Primary 53C25.

Key words and phrases. the total scalar curvature, critical point metric, Einstein.

First author was supported by Basic Science Research Program through the National Research Foundation of Korea(NRF) funded by the Ministry of Education, Science and Technology(2011-0005235), the second author by the Ministry of Education, Science and Technology(2011-0005211), and the third author by the Ministry of Education, Science and Technology(2011-0007465). 
critical point equation ( $\mathrm{CPE}$, hereafter):

$$
z_{g}=s_{g}^{*}(f)
$$

for some smooth function $f$ with vanishing mean value. Here, the operator $s_{g}^{\prime *}$ is given by

$$
s_{g}^{*}(f)=D_{g} d f-g \Delta_{g} f-f r_{g},
$$

where $D_{g} d f$ is the Hessian of $f, r_{g}$ is Ricci curvature of $g$, and $\Delta_{g}$ is the negative Laplacian of $g$. It is obvious that a solution $g$ of CPE is Einstein if $f$ is trivial. Thus we concentrate the case when $(g, f)$ is a non-trivial solution of CPE.

There are some known results about Conjecture I with a non-trivial function $f$. For example, Lafontaine showed that Conjecture I holds if a solution $g$ of $\mathrm{CPE}$ is conformally flat $([12])$ and $\operatorname{ker} s_{g}^{* *} \neq 0$. The third author showed that Conjecture I holds if $f \geq-1([7])$. The geometric structure of a solution to CPE which is Einstein is known to be simple due to Obata, who showed that such a solution is isometric to a standard $n$-sphere ([13]). For more details, we refer [1] and [7].

In this paper, we first consider the case when the Ricci tensor of $g$ is parallel. Then we have the following answer of Conjecture I for $\nabla r_{g}=0$ case.

Theorem 1.1. Let $(M, g)$ be an n-dimensional compact Riemannian manifold, $n \geq 3$, and $f$ be a non-trivial solution of CPE. If its Ricci tensor is parallel, then $(M, g)$ is isometric to a standard sphere.

Remark 1.2. It is well known that there is a relation between parallel Ricci tensor and harmonic curvature. We say that $(M, g)$ has harmonic curvature if $\delta R=0$. It turns out that every manifold with parallel Ricci tensor has harmonic curvature. However, there are examples of compact and noncompact Riemannian manifolds with $\delta R=0$ and $\nabla r_{g} \neq 0$ ([4], [6] Theorem 5.2). In virtue of Theorem 1.1, it is natural to ask whether a solution metric of CPE having harmonic curvature is Einstein. It is the topic of our forthcoming paper.

In the following we show that if $\delta R=0$ and $\nabla r_{g} \neq 0$, then $g$ is not necessarily a solution of CPE in general.

Theorem 1.3. Let $(M, g)$ be a compact, analytic $n$-dimensional manifold with harmonic curvature. If the Ricci tensor is not parallel and has less than three distinct eigenvalues at each point, $g$ cannot be a solution of CPE.

By a classification result in [4] (Theorem 2), the Riemannian covering of such a manifold $M$ in Theorem 1.3 is isometric to a warped product. Therefore, Theorem 1.3 is an immediate consequence of the following result.

Theorem 1.4. Let $g$ be a solution of CPE with a non-trivial function $f$. Then $(M, g)$ cannot be a warped product.

Recently, Lafontaine proved the following result. 
Theorem 1.5. Let $(M, g)$ be a compact 3-dimensional Riemannian manifold such that $\operatorname{dim} \operatorname{ker} s_{g}^{\prime *} \geq 2$. Then $(M, g)$ is isometric to a standard product $S^{1} \times S^{2}, S^{1} \times P^{2} \mathbb{R}$ or to the standard 3-sphere.

As an immediate consequence of Theorem 1.4 and Theorem 1.5, we have the following important result:

Theorem 1.6. Let $(M, g)$ be a compact 3-dimensional Riemannian manifold such that $\operatorname{dim}$ ker $s_{g}^{\prime *} \geq 2$ and $g$ is a solution of CPE. Then $(M, g)$ is isometric to the standard 3-sphere.

Remark 1.7. Fisher and Marsden suggested a F-M conjecture in [5], stating that if there exists a non-trivial function $f \in C^{\infty}(M)$ such that

$$
s_{g}^{\prime *}(f)=0,
$$

then $(M, g)$ is isometric to a standard sphere. It is known that $s_{g}$ is a nonnegative constant if ker $s_{g}^{\prime *} \neq 0([3],[5])$. It turns out that there are counterexamples of F-M conjecture, all of which are warped product ([10], [11]). Theorem 1.4 says that if $g$ is a warped product metric, then it cannot be a counterexample to Conjecture I, contrary to F-M conjecture case. In [8] and [9], this result has been proved when the dimension is 3 and 4 , while Theorem 1.4 holds for arbitrary dimension.

The paper is organized as follows. In Section 2, we shall give a brief collection of notations and some technical results. Sections 3 and 4 will be devoted to the proof of Theorem 1.4 which is crucially needed to prove our main results Theorem 1.1 and Theorem 1.3. In Section 5, by using Theorem 1.3, we will prove Theorem 1.1.

\section{Preliminaries}

Note that, by (1) and (2) the CPE may be written as

$$
(1+f) z_{g}=D_{g} d f+\frac{s_{g} f}{n(n-1)} g .
$$

Taking the trace of (4) gives

$$
\Delta_{g} f=-\frac{s_{g}}{n-1} f
$$

Here the scalar curvature $s_{g}$ is constant from the assumption.

For the proof of Theorem 1.4, we consider the case when $M$ is an $n$-dimensional warped product $M=B \times_{\psi^{2}} F$, where $(B, \breve{g})$ and $(F, \hat{g})$ are two Riemannian manifolds of dimension $n-p$ and $p$, respectively, and $\psi \in C^{\infty}(B)$ is a positive function. The metric $g$ is given by $g=\pi^{*} \check{g}+\psi^{2} \sigma^{*} \hat{g}$, where $*$ denotes the pull back, and $\pi$ and $\sigma$ denote the projections of $B \times F$ onto $B$ and $F$, respectively. Let

$\check{r}, \check{s}$ : the lifts to $M$ of Ricci and scalar curvature of $B$, respectively,

$\hat{r}, \hat{s}$ : the Ricci and scalar curvature of $F$, respectively, 
$X_{i}$ : a lifted horizontal orthonormal frame field, $i=1, \ldots, \operatorname{dim} B$,

$U_{j}$ : a lifted vertical orthonormal frame field, $j=1, \ldots, \operatorname{dim} F$,

$\mathcal{V}$ : the vertical distribution.

Then the following results are well-known.

Proposition 2.1 ([1]). We have

$$
\begin{aligned}
& r\left(X_{i}, X_{j}\right)=\check{r}\left(\check{X}_{i}, \check{X}_{j}\right)-\frac{p}{\psi} \check{D} d \psi\left(\check{X}_{i}, \check{X}_{j}\right), \\
& r\left(U_{i}, U_{j}\right)=\hat{r}\left(U_{i}, U_{j}\right)+\left\langle U_{i}, U_{j}\right\rangle\left(-\frac{\check{\Delta} \psi}{\psi}-(p-1) \frac{|d \psi|^{2}}{\psi^{2}}\right), \\
& s_{g}=\check{s}+\frac{\hat{s}}{\psi^{2}}-2 p \frac{\check{\Delta} \psi}{\psi}-p(p-1) \frac{|d \psi|^{2}}{\psi^{2}} .
\end{aligned}
$$

Corollary 2.2. If the scalar curvature $s_{g}$ of $M$ is constant, then the scalar curvature $\hat{s}$ of $F$ is constant.

Proof. For $p=n-1$, Proposition 2.1 gives

$$
\hat{s}=\psi^{2} s_{g}+(n-1)\left(2 \psi^{\prime \prime} \psi+(n-2) \psi^{\prime 2}\right) .
$$

Since $\hat{s}$ is a function on $F$, and the right-hand side of this equation is a function on $B, \hat{s}$ should be a constant function on $F$, i.e., $F$ is of constant scalar curvature. For $1<p<n-1$, this corollary follows similarly, since we have $\hat{s}=\psi^{2}\left(s_{g}-\check{s}\right)+2 p \psi \check{\Delta} \psi+p(p-1)|d \psi|^{2}$. Finally, $\hat{s}=0$ if $p=1$.

\section{Proof of Theorem 1.4}

Throughout the section we assume that $(M, g)$ is an $n$-dimensional compact warped product and $g$ is a solution of CPE with a non-trivial solution function $f$. The proof of Theorem 1.4 will be completed if there exists a contradiction.

Let $(M, g)=(B, \check{g}) \times_{\psi^{2}}(F, \hat{g})$ with $p=\operatorname{dim} F$, and denote $s$ for $s_{g}$. In the following we break into three cases: Case $p=1$ (Subsection 3.1), Case $p=n-1$ (Subsection 3.2), Case $1<p<n-1$ (Subsection 3.3). We will derive a contradiction in each cases.

\subsection{Case $p=1$}

By the definition of the Laplacian

$$
\left\langle D_{U} d f, U\right\rangle=\Delta f-\check{\Delta} f=-\frac{s}{n-1} f-\check{\Delta} f
$$

and by (4) and Proposition 2.1,

$$
(1+f)\left(-\frac{\check{\Delta} \psi}{\psi}-\frac{s}{n}\right)=\left\langle D_{U} d f, U\right\rangle+\frac{s}{n(n-1)} f .
$$

Combining (7) and (8), we have

$$
(1+f) \check{\Delta} \psi+\frac{s}{n} \psi=\psi \check{\Delta} f .
$$

Integrating both sides of (9) over $B$, we obtain

$$
\int_{B} f \check{\Delta} \psi+\frac{s}{n} \int_{B} \psi=\int_{B} \psi \check{\Delta} f=\int_{B} f \check{\Delta} \psi,
$$


which implies that

This is a contradiction since $\psi>0$

$$
\int_{B} \psi=0
$$

\subsection{Case $p=n-1$}

First we prove that $f \in C^{\infty}(B)$. From (4)

$$
0=(1+f) z\left(X, U_{i}\right)=\left\langle D_{X} d f, U_{i}\right\rangle=X\left\langle d f, U_{i}\right\rangle-\left\langle d f, D_{X} U_{i}\right\rangle
$$

for a lifted horizontal vector field $X$. Note that $U_{i}=\frac{1}{\psi} \widehat{U_{i}}$, where $\widehat{U_{i}}$ is a lift of vector field on $F$. Therefore

$$
D_{X} U_{i}=D_{X}\left(\frac{1}{\psi} \widehat{U_{i}}\right)=-\frac{X(\psi)}{\psi^{2}} \widehat{U_{i}}+\frac{1}{\psi} D_{X} \widehat{U_{i}}=0,
$$

where we used the fact that $D_{X} \widehat{U_{i}}=\frac{X(\psi)}{\psi} \widehat{U_{i}}$ in the last equality (cf. [14]). Now, substitution of (11) into (10) gives

$$
X U_{i}(f)=X\left\langle d f, U_{i}\right\rangle=0 .
$$

Therefore $U_{i}(f)=\frac{1}{\psi} \widehat{U_{i}}(f) \in C^{\infty}(F)$, and it is easy to see that $f$ can be written as

$$
f=\psi b+c,
$$

where $b \in C^{\infty}(F)$ and $c=c(t) \in C^{\infty}(B)$. Substituting (13) into $(1+f) z(X, X)=\left\langle D_{X} d f, X\right\rangle+\frac{s f}{n(n-1)}$ gives

$$
(1+\psi b+c)\left(-(n-1) \frac{\psi^{\prime \prime}}{\psi}-\frac{s}{n}\right)=\psi^{\prime \prime} b+c^{\prime \prime}+\frac{s}{n(n-1)}(\psi b+c),
$$

where we used Proposition 2.1 and the fact that, from $\check{d f}=b d \psi+d c$ and $D_{X} \widehat{d f}=\mathcal{V} D_{X} \widehat{d f}$,

$$
\begin{aligned}
\left\langle D_{X} d f, X\right\rangle & =\left\langle D_{X} \check{d f}, X\right\rangle+\left\langle D_{X} \widehat{d f}, X\right\rangle=\left\langle D_{X} \check{d f}, X\right\rangle \\
& =b\left\langle D_{X} d \psi, X\right\rangle+\left\langle D_{X} d c, X\right\rangle=\psi^{\prime \prime} b+c^{\prime \prime} .
\end{aligned}
$$

Thus, the equation (14) can be rewritten as

$$
b\left(-n \psi^{\prime \prime}-\frac{s}{n-1} \psi\right)=(1+c)\left(\frac{(n-1) \psi^{\prime \prime}}{\psi}+\frac{s}{n}\right)+c^{\prime \prime}+\frac{s}{n(n-1)} c .
$$

Note that both $-n \psi^{\prime \prime}-\frac{s}{n-1} \psi$ and the right-hand side are functions of $B$, while $b \in C^{\infty}(F)$. Thus, in order that the equation (15) holds for any $t$, either $b$ is constant or $-n \psi^{\prime \prime}-\frac{s}{n-1} \psi$ has to be zero.

We show that $b$ must be a constant. On the contrary, suppose that $b$ is not a constant. Then $-n \psi^{\prime \prime}-\frac{s}{n-1} \psi=0$. Since $B$ is complete, $\psi$ has to be defined on the whole of $\mathbb{R}$. Moreover, since $B=S^{1}, \psi$ has to be periodic. Therefore

$$
0=\int_{B}\left(\frac{\psi^{\prime}}{\psi}\right)^{\prime}=\int_{B} \frac{\psi^{\prime \prime}}{\psi}-\frac{\psi^{2}}{\psi^{2}}=-\int_{B}\left(\frac{s}{n(n-1)}+\frac{\psi^{2}}{\psi^{2}}\right)<0 .
$$


This contradiction leads to the conclusion that $b$ is constant, and in virtue of (13) $f \in C^{\infty}(B)$.

Now we prove that $g$ cannot be a solution of CPE. Since $f \in C^{\infty}(B)$, we compute

$$
\left\langle D_{U_{i}} d f, U_{i}\right\rangle=\left\langle D_{U_{i}} \check{d f}, U_{i}\right\rangle=\frac{\psi^{\prime} f^{\prime}}{\psi}
$$

for each $i$, since $D_{U_{i}} \check{d f}=\frac{\psi^{\prime} \partial_{t} f}{\psi} U_{i}$ (cf. [1]). Now, from (4)

$$
(1+f)\left(\hat{r}\left(U_{i}, U_{i}\right)-\frac{\psi^{\prime \prime}}{\psi}-(n-2) \frac{\psi^{\prime 2}}{\psi^{2}}-\frac{s}{n}\right)=\frac{\psi^{\prime}}{\psi} f^{\prime}+\frac{s f}{n(n-1)} .
$$

Then it is easy to see that $\hat{r}\left(U_{i}, U_{i}\right)=\hat{r}\left(U_{j}, U_{j}\right)$ for $i \neq j$. Thus $\hat{r}\left(\widehat{U_{i}}, \widehat{U_{i}}\right)=\frac{\hat{s}}{n-1}$ for all $1 \leq i \leq n-1$, and $\hat{r}\left(U_{i}, U_{j}\right)=0$ for $i \neq j$. In other words, $F$ is an Einstein manifold and $\hat{r}\left(U_{i}, U_{i}\right)=\frac{\hat{s}}{(n-1) \psi^{2}}$.

On the other hand, for any Riemannian manifold the divergence $\delta R$ of its curvature tensor $R$ satisfies the well-known identity

$$
\delta R=-d r_{g},
$$

where $d r_{g}$ denotes the Riemannian exterior derivative of $r_{g}$. The local coordinate expression for (18) is

$$
\nabla^{i} R_{a i b c}=\nabla_{c} r_{a b}-\nabla_{b} r_{a c}
$$

If we make the change of the function $\zeta=\psi^{\frac{n}{2}}$ in (6), we have

$$
\zeta^{\prime \prime}-\frac{n \hat{s}}{4(n-1)} \zeta^{1-\frac{4}{n}}=-\frac{n s}{4(n-1)} \zeta .
$$

Derdziński showed in Lemma 1 of [4] that if $\psi$ is non-constant and $n \geq 3$, then $I \times \psi_{\psi^{2}} F$ has harmonic curvature if and only if $(F, \hat{g})$ is an Einstein space and the positive function $\zeta=\psi^{\frac{n}{2}}$ on $I$ satisfies (20). Recall that $M$ has harmonic curvature if and only if $\delta R=0$. Here $M$ is analytic in a suitable local coordinate ([2]) and is not conformally flat unless $\hat{g}$ has constant sectional curvature (see Remark 3.1).

Thus we may conclude that $M$ has harmonic curvature, and by (18) and (19), $\nabla_{c} r_{a b}=\nabla_{b} r_{a c}$. In particular, for $i \geq 2$ we have $\nabla_{1} r_{i i}=0$. Note that by Proposition 2.1 and (6)

$$
r_{i i}=r\left(U_{i}, U_{i}\right)=\frac{\hat{s}}{(n-1) \psi^{2}}-\frac{\psi^{\prime \prime}}{\psi}-(n-2) \frac{\psi^{\prime 2}}{\psi^{2}}=\frac{s}{n-1}+\frac{\psi^{\prime \prime}}{\psi}
$$

for $i \geq 2$. Since the equation (6) together with the fact that $\hat{s}$ is constant implies

$$
\frac{\psi^{\prime \prime \prime}}{\psi}=-\frac{s}{n-1} \frac{\psi^{\prime}}{\psi}-(n-1) \frac{\psi^{\prime \prime} \psi^{\prime}}{\psi^{2}},
$$


it follows from (21) and (22) that

$$
0=\nabla_{1} r_{i i}=-\frac{\psi^{\prime}}{\psi}\left(\frac{s}{n-1}+n \frac{\psi^{\prime \prime}}{\psi}\right) .
$$

Therefore, when $\psi^{\prime} \neq 0$, by $(23)$

$$
\frac{\psi^{\prime \prime}}{\psi}=-\frac{s}{n(n-1)}<0 .
$$

By the analytic property and continuity of $\psi$, the equation (24) holds on all of $B=S^{1}$. However, if $\psi$ takes its minimum, $\frac{\psi^{\prime \prime}}{\psi} \geq 0$, which is impossible. This contradiction completes the proof.

Remark 3.1. When $n=3,4$, the proof can be simplified as follows. Since $\hat{g}$ is Einstein, $(F, \hat{g})$ has constant curvature. Thus $(M, g)$ is conformally flat, and is Einstein by Lafontaine [12], and is isometric to $S^{n}$ by Obata [13], as mentioned in Introduction. Hence a warped product $S^{1} \times_{\psi^{2}} F$ cannot be a solution of CPE.

\subsection{Case $1<p<n-1$}

First we need the following lemma, which will be proved in Section 4 .

Lemma 3.2. If $f \in C^{\infty}(B)$, then $g$ cannot be a solution of $C P E$.

Once we assume Lemma 3.2, it suffices to prove that $f \in C^{\infty}(B)$. First, as in Case $p=n-1$, we have

$$
f=\psi b+c,
$$

where $b \in C^{\infty}(F)$ and $c \in C^{\infty}(B)$. By Proposition 2.1 and (4),

$$
(1+f)\left(\check{s}-\frac{p}{\psi} \check{\Delta} \psi-\frac{n-p}{n} s\right)=\sum_{i=1}^{n-p}\left\langle D_{X_{i}} d f, X_{i}\right\rangle+\frac{(n-p) s f}{n(n-1)} .
$$

Now, substitution (25) into (26) gives

$$
(1+\psi b+c)\left(\check{s}-\frac{p}{\psi} \check{\Delta} \psi-\frac{n-p}{n} s\right)=b \check{\Delta} \psi+\check{\Delta} c+\frac{(n-p) s}{n(n-1)}(\psi b+c),
$$

where we used the fact that

$$
\begin{aligned}
\sum_{i=1}^{n-p}\left\langle D_{X_{i}} d f, X_{i}\right\rangle & =\sum_{i=1}^{n-p}\left(\left\langle D_{X_{i}} \check{d f}, X_{i}\right\rangle+\left\langle D_{X_{i}} \widehat{d f}, X_{i}\right\rangle\right)=\sum_{i=1}^{n-p}\left\langle D_{X_{i}} \check{d f}, X_{i}\right\rangle \\
& =b \sum_{i=1}^{n-p}\left\langle D_{X_{i}} d \psi, X_{i}\right\rangle+\sum_{i=1}^{n-p}\left\langle D_{X_{i}} d c, X_{i}\right\rangle=b \check{\Delta} \psi+\check{\Delta} c
\end{aligned}
$$

with $D_{X_{i}} \hat{d f}=\mathcal{V} D_{X_{i}} \hat{d f}$. The equation (27) can be rewritten as

$$
b\left(\psi \check{s}-(p+1) \check{\Delta} \psi-\frac{n-p}{n-1} s \psi\right)=\check{\Delta} c-(c+1) \Theta+\frac{(n-p) s}{n(n-1)} c,
$$


where $\Theta=\check{s}-\frac{p}{\psi} \check{\Delta} \psi-\frac{n-p}{n} s$. Note that both $\psi \check{s}-(p+1) \check{\Delta} \psi-\frac{n-p}{n-1} s \psi$ and the right-hand side of (28) are functions of $B$, while $b \in C^{\infty}(F)$. Thus, in order that the equation (28) holds, either $b$ is constant or $\psi \check{s}-(p+1) \check{\Delta} \psi-\frac{n-p}{n-1} s \psi$ has to be zero.

If we prove that $b$ is constant, we are done by (25). Assume that $b$ is not constant. Then we have

$$
\check{s}=(p+1) \frac{\check{\Delta} \psi}{\psi}+\frac{n-p}{n-1} s,
$$

and

$$
\check{\Delta} c-(c+1)\left(\check{s}-p \frac{\check{\Delta} \psi}{\psi}-\frac{n-p}{n} s\right)+\frac{(n-p) s}{n(n-1)} c=0 .
$$

Substitution of (29) into (30) gives

$$
\psi \check{\Delta} c-(c+1) \check{\Delta} \psi-\frac{n-p}{n(n-1)} s \psi=0 .
$$

Now, integrating both sides of (31) over $B$, we have

$$
s \frac{n-p}{n(n-1)} \int_{B} \psi=0
$$

which contradicts the fact that $\psi>0$.

\section{Proof of Lemma 3.2}

Assume that $g$ is a warped product satisfying (4) and $f \in C^{\infty}(B)$. In the following, we shall prove that this assumptions give a contradiction. Thus we may conclude that $g$ cannot be a solution of CPE, proving Lemma 3.2.

Consider a set $H=\{x \in B \mid f(x)=-1\}$. Here $H$ should be non-empty, otherwise by Main Theorem in [7] $(M, g)$ is isometric to a standard sphere $S^{n}$, which is not a warped product.

Lemma 4.1. For a unit tangent vector field $X \in T B$ to $H$,

$$
D_{X} X=-\frac{s}{n(n-1)} d f+(\text { tangential terms }) \quad \text { on } H \text {. }
$$

Proof. It is shown in [7] that a point of $H$, which is a critical point of $f$, is a non-degenerate local minimum point of $f$, and that such non-degenerate critical points are isolated. Therefore, $H$ is a set consisting of finite critical points of $f$, or hypersurfaces of $M$, or union of both.

Putting $W=|d f|^{2}$, it was proved in [7] that $W$ is constant in each component of $H$ and does not vanish on $H$. Therefore, in a small tubular neighborhood of $H$, we may take orthonormal frame fields $\left\{X_{1}, \ldots, X_{n-p-1}, N\right\}$, where $N=$ $\frac{d f}{W^{\frac{1}{2}}}$ on $H$. From (4), we have

$$
D d f=\frac{s}{n(n-1)} g
$$


on $H$. Then, it follows that on $H$ we have

$$
\frac{s}{n(n-1)}=\left\langle D_{X_{i}} d f, X_{i}\right\rangle=X_{i}\left\langle d f, X_{i}\right\rangle-\left\langle d f, D_{X_{i}} X_{i}\right\rangle=-\left\langle d f, D_{X_{i}} X_{i}\right\rangle .
$$

Hence, $D_{X_{i}} X_{i}=-\frac{s}{n(n-1)} \frac{1}{W^{\frac{1}{2}}} N+($ tangential terms) on $H$, which is (33).

Now we are ready to prove Lemma 3.2.

Proof of Lemma 3.2. In virtue of Proposition 2.1, (4) may be reduced to

$$
(1+f)\left(\check{s}-\frac{p}{\psi} \check{\Delta} \psi-\frac{s}{n}(n-p)\right)=\check{\Delta} f+\frac{(n-p) s f}{n(n-1)},
$$

and

$$
(1+f)\left(\frac{\hat{s}}{p \psi^{2}}-\frac{\check{\Delta} \psi}{\psi}-(p-1) \frac{|d \psi|^{2}}{\psi^{2}}-\frac{s}{n}\right)=\frac{\langle d \psi, d f\rangle}{\psi}+\frac{s f}{n(n-1)},
$$

where $\check{g}(d \psi, d f)=\langle d \psi, d f\rangle$. Hence, using (35) and (36) we have

$$
\check{\Delta} f=\frac{(n-p) s}{n(n-1)},
$$

and

$$
\frac{\langle d \psi, d f\rangle}{\psi}=\frac{s}{n(n-1)}
$$

on $H$. The relation $\sum_{i=1}^{n-p} z\left(X_{i}, X_{i}\right)+\sum_{i=1}^{p} z\left(U_{i}, U_{i}\right)=0$ gives

$$
(1+f)\left(-\check{s}+\frac{p}{\psi} \check{\Delta} \psi+\frac{s}{n}(n-p)\right)=p \frac{\langle d \psi, d f\rangle}{\psi}+p \frac{s f}{n(n-1)} .
$$

In virtue of (34) and (38)

$$
\frac{\left\langle d \psi, D_{d f} d f\right\rangle}{\psi}=\frac{s}{n(n-1)} \frac{\langle d f, d \psi\rangle}{\psi}=\left(\frac{s}{n(n-1)}\right)^{2}=\frac{\langle d \psi, d f\rangle^{2}}{\psi^{2}} .
$$

So taking the Lie derivative of (39) with respect to $d f$ on $H$, we have

$$
\begin{aligned}
& W\left(-\check{s}+\frac{p}{\psi} \check{\Delta} \psi+\frac{s}{n}(n-p)\right) \\
= & p \frac{\left\langle D_{d f} d \psi, d f\right\rangle}{\psi}+p \frac{\left\langle d \psi, D_{d f} d f\right\rangle}{\psi}-p \frac{\langle d \psi, d f\rangle^{2}}{\psi^{2}}+\frac{s p}{n(n-1)} W \\
= & p \frac{\left\langle D_{N} d \psi, N\right\rangle}{\psi} W+\frac{s p}{n(n-1)} W .
\end{aligned}
$$

Therefore

$$
\check{s}=s\left(\frac{n-p-1}{n-1}\right)+\frac{p}{\psi} \sum_{i=1}^{n-p-1}\left\langle D_{X_{i}} d \psi, X_{i}\right\rangle .
$$


On the other hand, in order to calculate $z(X, X)$ we take the Lie derivative of (4) with respect to $d f$ on $H$. Then on $H$

$$
\begin{aligned}
W z(X, X) & =\left\langle D_{d f} D_{X} d f, X\right\rangle+\left\langle D_{X} d f, D_{d f} X\right\rangle+\frac{s}{n(n-1)} W \\
& =W^{\frac{1}{2}}\left\langle D_{N} D_{X} d f, X\right\rangle+\left|D_{X} d f\right|^{2}+\frac{s}{n(n-1)} W \\
& =W^{\frac{1}{2}}\left\langle D_{N} D_{X} d f, X\right\rangle+\frac{s^{2}}{n^{2}(n-1)^{2}}+\frac{s}{n(n-1)} W,
\end{aligned}
$$

where we used the fact that $W$ is constant on $H, D_{d f} X=W^{-\frac{1}{2}} D_{N} X=$ $W^{-\frac{1}{2}} D_{X} N=D_{X} d f$, and $D_{X} d f=\frac{s}{n(n-1)} X$ on $H$. However, the relation $D_{N} D_{X} d f=D_{X} D_{N} d f+R(X, N) d f$ gives

$$
\begin{aligned}
\left\langle D_{N} D_{X} d f, X\right\rangle & =\left\langle D_{X} D_{N} d f, X\right\rangle+\langle R(X, N) d f, X\rangle \\
& =X\left\langle D_{N} d f, X\right\rangle-\left\langle D_{N} d f, D_{X} X\right\rangle-W^{\frac{1}{2}} K(X, N) \\
& =\frac{s}{n(n-1) W^{\frac{1}{2}}}\left\langle D_{N} d f, N\right\rangle-W^{\frac{1}{2}} K(X, N) \\
& =\frac{s^{2}}{n^{2}(n-1)^{2} W^{\frac{1}{2}}}-W^{\frac{1}{2}} K(N, N),
\end{aligned}
$$

where we used the fact that $\left\langle D_{N} d f, X\right\rangle=0$, (33), and (34) on $H$. Now substituting (42) into (41), we have

$$
z(X, X)=\frac{2 s^{2}}{n^{2}(n-1)^{2} W}-K(X, N)+\frac{s}{n(n-1)} .
$$

Thus

$$
\sum_{i=1}^{n-p-1} z\left(X_{i}, X_{i}\right)=\frac{2 s^{2}(n-p-1)}{n^{2}(n-1)^{2} W}-\check{r}(N, N)+\frac{s}{n(n-1)}(n-p-1),
$$

where we used the fact that $\sum_{i=1}^{n-p-1} K\left(X_{i}, N\right)=\check{r}(N, N)$.

Note that from Proposition 2.1 we have

$$
\sum_{i=1}^{n-p-1} z\left(X_{i}, X_{i}\right)=\check{s}-\check{r}(\check{N}, \check{N})-\sum_{i=1}^{n-p-1} \frac{p}{\psi} \check{D} d \psi\left(\check{X}_{i}, \check{X}_{i}\right)-\frac{s}{n}(n-p-1) .
$$

Equating (43) with (44) gives

$$
\check{s}=\frac{2 s^{2}(n-p-1)}{n^{2}(n-1)^{2} W}+s\left(\frac{n-p-1}{n-1}\right)+\frac{p}{\psi} \sum_{i=1}^{n-p-1}\left\langle D_{X_{i}} d \psi, X_{i}\right\rangle .
$$

The equation (45) clearly contradicts the equation (40).

Remark 4.2. This proof is not applicable for Case $p=1$ since the equation (40) is meaningful only for $p \geq 2$. 


\section{Proof of Theorem 1.1}

In this section we will prove Theorem 1.1. It suffices to prove the following lemma, since if $M$ is Einstein, it is isometric to a standard sphere by Obata $([13])$.

Lemma 5.1. If $g$ is a solution of $C P E$ with $\nabla r_{g}=0$, then $(M, g)$ is Einstein.

Proof. If $\nabla r_{g}=0$, from the fact that Ricci curvature remains invariant under the action by the holonomy groups of $M$, and the de Rham decomposition theorem, $M$ is locally isometric to products of Einstein manifolds ([6]), $M_{1} \times$ $M_{2} \times \cdots \times M_{k}$. Denote $M_{2} \times \cdots \times M_{k}$ by $F$. Thus there exists a smooth map $\varphi:(M, g) \rightarrow\left(M_{1} \times F, \tilde{g}\right)$ such that for every $p \in M$ there exists a neighborhood $U$ for which $\left.\varphi\right|_{U}: U \rightarrow \varphi(U)$ is an isometry. Then $\tilde{g}=\left(\varphi^{-1}\right)^{*} g$ with $\tilde{f}=f \circ \varphi^{-1}$ satisfies

$$
(1+\tilde{f}) z_{\tilde{g}}=D_{\tilde{g}} d \tilde{f}+\frac{\tilde{s} \tilde{f}}{n(n-1)} \tilde{g} .
$$

We will follow the proof of Theorem 1.4.

Let $p=\operatorname{dim} F$. Without loss of generality, we can assume that $\operatorname{dim} M_{1} \geq 1$. We will derive a contradiction when $1 \leq p \leq n-1$, implying that $p=0$, i.e., $M$ is itself locally isometric to an Einstein manifold, proving our lemma. Note that $\psi \equiv 1$, and $\check{s}$ and $\hat{s}$ for $\tilde{g}$ are constants.

For $p=1$, from $\left\langle D_{U} d \tilde{f}, U\right\rangle=-\frac{\tilde{s}}{n-1} \tilde{f}-\check{\Delta} \tilde{f}$ and

$$
-\frac{\tilde{s}}{n}(1+\tilde{f})=\left\langle D_{U} d \tilde{f}, U\right\rangle+\frac{\tilde{s}}{n(n-1)} \tilde{f}
$$

we obtain

$$
\check{\Delta} \tilde{f}=\frac{\tilde{s}}{n}>0 .
$$

However, $\check{\Delta} \tilde{f} \leq 0$ at a local maximum point of $\tilde{f}$, which is impossible.

For $p=n-1$, following the first part of the proof in Section $3.2, \tilde{f}=b+c$, where $b \in C^{\infty}(F)$ and $c \in C^{\infty}\left(M_{1}\right)$. By (15) we have

$$
-\frac{\tilde{s}}{n-1} b=\frac{\tilde{s}}{n}+c^{\prime \prime}+\frac{\tilde{s}}{n-1} c .
$$

Thus $b$ is constant and $\tilde{f}=b+c \in C^{\infty}\left(M_{1}\right)$. Now from (6) and (17), $\hat{s}=\tilde{s}$ and

$$
(1+\tilde{f})\left(\frac{\hat{s}}{n-1}-\frac{\tilde{s}}{n}\right)=\frac{\tilde{s} \tilde{f}}{n(n-1)},
$$

which implies that $\frac{\tilde{s}}{n(n-1)}=0$, a contradiction.

For $1<p<n-1$, following the first part of the proof in Section 3.3, $\tilde{f}=b+c$ and by (28)

$$
b\left(\check{s}-\frac{n-p}{n-1} \tilde{s}\right)=\check{\Delta} c-(c+1)\left(\check{s}-\frac{n-p}{n} \tilde{s}\right)-\frac{n-p}{n(n-1)} c .
$$


If $\check{s} \neq \frac{n-p}{n-1} \tilde{s}$, then $b$ is constant and $\tilde{f}=b+c \in C^{\infty}\left(M_{1}\right)$. Then from (36)

$$
(1+\tilde{f})\left(\frac{\hat{s}}{p}-\frac{\tilde{s}}{n}\right)=\frac{\tilde{s}}{n(n-1)} \tilde{f},
$$

which is simplified as

$$
\left(\frac{\tilde{s}}{n-1}-\frac{\hat{s}}{p}\right) \tilde{f}=\frac{\hat{s}}{p}-\frac{\tilde{s}}{n}
$$

In order for $(47)$ to hold, the following equations should hold:

$$
\frac{\tilde{s}}{n-1}-\frac{\hat{s}}{p}=0=\frac{\hat{s}}{p}-\frac{\tilde{s}}{n},
$$

which implies that $\frac{\tilde{s}}{n(n-1)}=0$, a contradiction.

$$
\text { If } \check{s}=\frac{n-p}{n-1} \tilde{s} \text {, by }(26)
$$

$$
\frac{(n-p) \tilde{s}}{n(n-1)}(1+\tilde{f})=\check{\Delta} \tilde{f}+\frac{(n-p) \tilde{s}}{n(n-1)} \tilde{f},
$$

which is simplified as

$$
\check{\Delta} \tilde{f}=\frac{(n-p) \tilde{s}}{n(n-1)}>0 .
$$

However, $\check{\Delta} \tilde{f} \leq 0$ at a local maximum point of $\tilde{f}$, which is impossible.

\section{References}

[1] A. L. Besse, Einstein Manifolds, Springer-Verlag, Berlin, Heidelberg, 1987.

[2] R. L. Bishop and B. O'Neill, Manifolds of negative curvature, Trans. Amer. Math. Soc. 145 (1969), 1-49.

[3] J. P. Bourguignon, Une stratification de l'espace des structures riemanniennes, Compositio Math. 30 (1975), 1-41.

[4] A. Derdziński, On compact Riemannian manifolds with harmonic curvature, Math. Ann. 259 (1982), no. 2, 145-152.

[5] A. E. Fischer and J. E. Marsden, Manifolds of Riemannian metrics with prescribed scalar curvature, Bull. Amer. Math. Soc. 80 (1974), 479-484.

[6] A. Gray, Einstein-like manifolds which are not Einstein, Geom. Dedicata 7 (1978), no. $3,259-280$.

[7] S. Hwang, Critical points of the total scalar curvature functional on the space of metrics of constant scalar curvature, Manuscripta Math. 103 (2000), no. 2, 135-142.

[8] S. Hwang and J. W. Chang, Critical points and warped product metrics, Bull. Korean Math. Soc. 41 (2004), no. 1, 117-123.

[9] , The Critical point equation on a four dimensional warped product manifold, Bull. Korean Math. Soc. 43 (2006), no. 4, 679-692.

[10] O. Kobayashi, A differential equation arising from scalar curvature function, J. Math. Soc. Japan 34 (1982), no. 4, 665-675.

[11] J. Lafontaine, Remarques sur les variétés conformément plates, Math. Ann. 259 (1982), no. 3, 313-319.

[12] _ Sur la géométrie d'une généralisation de l'équation différentielle d'Obata, J. Math. Pures Appl. (9) 62 (1983), no. 1, 63-72.

[13] M. Obata, Certain conditions for a Riemannian manifold to be isometric with a sphere, J. Math. Soc. Japan 14 (1962), no. 3, 333-340. 
[14] B. O'Neill, Semi-Riemannian Geometry, Academic Press, New York, 1983.

JEONGWOOK CHANG

Department of Mathematics Education

DANKOOK UNIVERSITY

YONGIN 448-701, KorEA

E-mail address: jchang@dankook.ac.kr

Seungsu Hwang

Department of Mathematics

Chung-Ang University

SEOUl 156-756, KoreA

E-mail address: seungsu@cau.ac.kr

GABJIN YuN

Department of Mathematics

MYONG Ji UNIVERSITY

Yongin 449-728, KoreA

E-mail address: gabjin@mju.ac.kr 